\title{
Effects of Systemic Glucocorticoid Use on Fracture Risk: A Population-Based Study
}

\author{
Ji Weon Koh ${ }^{1, *}$, Junkang Kim ${ }^{2, *}$, Hyemin $\mathrm{Cho}^{3}$, Yong-Chan $\mathrm{Ha}^{4}$, Tae-Young Kim ${ }^{5}$, Young-Kyun Lee ${ }^{6}$, Ha Young Kim \\ Sunmee Jang ${ }^{3}$ \\ ${ }^{1}$ Inhu-Chongno Pharmacy, Jeonju; ${ }^{2}$ Department of Pharmacy, Mediplex Sejong Hospital; ${ }^{3}$ College of Pharmacy and Gachon \\ Institute of Pharmaceutical Sciences, Gachon University, Incheon; ${ }^{4}$ Department of Orthopaedic Surgery, Chung-Ang University \\ College of Medicine; ${ }^{5}$ Department of Orthopaedic Surgery, Konkuk University Medical Center, Konkuk University School of \\ Medicine, Seoul; ${ }^{6}$ Department of Orthopaedic Surgery, Seoul National University Bundang Hospital, Seongnam; ${ }^{7}$ Department of \\ Internal Medicine, Gangneung Asan Hospital, University of Ulsan College of Medicine, Gangneung, Korea
}

Background: Long-term glucocorticoid use increases fracture risk by reducing bone mass. This study evaluated the relationship between hip and vertebral fractures and the total amount of systematic glucocorticoid use.

Methods: We randomly selected 1,896,159 people aged 20 to 100 years who participated in the National Health Checkup program in 2006. The amount of glucocorticoids prescribed was calculated based on the defined daily dose (DDD). The total DDD was obtained by adding oral and parenteral glucocorticoids for 6 months from the index date. Subjects were categorized into four groups according to total glucocorticoid DDDs: non-users (DDDs $=0)$, low users $(0<$ DDDs $\leq 45)$, intermediate users $(45<$ DDDs $\leq 90)$, and high users $(90<$ DDDs). We followed them for 2 years. A multivariate Cox proportional hazard model was used to evaluate the effects of the total amount of glucocorticoid use on hip and vertebral fractures.

Results: Higher glucocorticoid use was associated with a higher risk of vertebral fracture. Relative to non-users, the vertebral fracture risk was 1.39 times higher in the low-user group, 1.94 times higher in the intermediate-user group, and 2.43 times higher in the highuser group. The risk of hip fracture was 1.72 times higher in intermediate users and 3.28 times higher in high users than in non-users. Conclusion: As the amount of glucocorticoid use for 6 months increased, the risk of hip and vertebral fractures became higher. In order to prevent fractures, it is necessary for doctors to evaluate the total amount of glucocorticoid prescribed to the patient and to provide appropriate treatment.

Keywords: Glucocorticoids; Fracture; Epidemiologic studies; Osteoporosis

Received: 24 March 2020, Revised: 1 June 2020, Accepted: 16 June 2020

Corresponding authors: Ha Young Kim

Department of Internal Medicine, Gangneung Asan Hospital, University of Ulsan College of Medicine, 38 Bangdong-gil, Gangneung 25440, Korea

Tel: +82-33-610-3114, Fax: +82-33-610-3900, E-mail: hykimmd@hanmail.net

Sunmee Jang

College of Pharmacy and Gachon Institute of Pharmaceutical Sciences, Gachon

University, Incheon, 191 Hambangmoe-ro, Yeonsu-gu, Incheon 21936, Korea

Tel: +82-32-820-4941, Fax: +82-32-820-4820, E-mail: smjang@gachon.ac.kr

\section{Copyright $\odot 2020$ Korean Endocrine Society}

This is an Open Access article distributed under the terms of the Creative Commons Attribution Non-Commercial License (https://creativecommons.org/ licenses/by-nc/4.0/) which permits unrestricted non-commercial use, distribution, and reproduction in any medium, provided the original work is properly cited.

*These authors contributed equally to this work. 


\section{INTRODUCTION}

Long-term glucocorticoids (GCs) treatment is known to be a major risk factor for osteoporotic fractures and the most common cause of secondary osteoporosis [1,2]. The effects of GCs on bone fragility primarily occur through their direct impact on osteoblasts, osteocytes, and osteoclasts; specifically, they decrease the production of osteoblasts and osteocytes, while prolonging the lifespan of osteoclasts [3]. Indirect effects include reduced muscle mass, decreased renal calcium resorption and levels of sex hormones, and alterations in parathyroid hormone pulsatility [4]. Bone loss in GC-induced osteoporosis (GIOP) is biphasic, with a reduction of bone mineral density (BMD) of $6 \%$ to $12 \%$ within the first year, followed by a slower annual loss of approximately $3 \%$ [5]. The risk of fracture increases rapidly within the first 3 months after initiation of GC therapy. The risk of all fractures was found to decline toward baseline rapidly after discontinuation of oral GCs [6].

The prevalence of oral GC use in the community population has been reported to be $0.85 \%$ in the United Kingdom population age $\geq 18$ years and $1.2 \%$ in the United States population age $\geq 20$ years. GC use increased with age and was around $2.5 \%$ in people over 60 years old $[7,8]$. However, little epidemiological data have been reported on the use of GCs in Korea. Our previous study reported about $1 \%$ of women over 50 years of age used oral GCs for more than 30 days a year using a randomly sample of the National Health Checkup Database [9].

The fracture risk assessment tool (FRAX), developed by the World Health Organization (WHO), includes the use of oral GCs as an independent risk factor for the prediction of fractures. In this model, the definition of GC use was initially ever-users based on a meta-analysis of seven cohorts [10]. However, the risk of fracture is largely determined by the dose and duration of GC use. Van Staa et al. [6] reported that the relative risk of vertebral and hip fractures increased depending on the daily GC dose using the General Practice Research Database (GPRD). Therefore, the risky level of oral GC use in the FRAX model is now assumed to be equivalent to $5 \mathrm{mg}$ daily or more of prednisolone for more than 3 months. Recently, the FRAX model with correction for daily GC usage has been used in the United King$\operatorname{dom}[11]$.

So far, most studies regarding GC use analyzed mainly oral $\mathrm{GC}$ and provided various indicators related to drug use such as mean daily dose, cumulative dose, duration and highest dose etc. The criteria presented in each study are different, so it can be confusing when applying them in real clinical practice. In addition, intermittent use of high-dose oral GCs may result in a small increased risk of osteoporotic fracture [12]. There are several reports of increased risk of fractures even when using injected or inhaled GCs $[13,14]$. Therefore, this study aimed to investigate the dose-dependent fracture risk based on the total amount of systemic $\mathrm{GC}$ use among general population. For drug usage evaluation, we used defined daily dose (DDD) that is the most regarded drug utilization metric and was developed as a universal measure of drug consumption. In order to be easily applied to clinical practice, the drug usage was evaluated for a period of time after the $\mathrm{GC}$ was first used.

\section{METHODS}

\section{Source of data}

The Korean National Health Insurance Service (NHIS) provides health insurance to all Koreans, and all Koreans are obliged to become members of this national insurance system. The Korean NHIS performs National Health Checkups every 2 years for insured Koreans over 40 years of age, and these checkups are enforced by law and performed by local hospitals that are fully qualified based on the NHIS criteria. The National Health Checkups include height, weight and blood pressure measurements, chest radiography, urinalysis, blood count, and blood chemistry. In addition, a self-administered questionnaire is used to collect information on patients' medical history, current health status, family history, tobacco and alcohol consumption, dietary preferences, and leisure-time physical activities.

\section{Study design}

Two million men and women were randomly selected from the participants who had received health checkups from January 1 to December 31, 2006. We excluded subjects aged $<20$ and $>100$ years $(n=3,759)$. In addition, subjects who had missing information for smoking, alcohol use, body mass index (BMI), and place of residence $(n=601)$ were excluded. There were no missing data for other variables. Subjects who died $(n=1,839)$ or suffered a fracture $(n=1,186)$ within 6 months after the index date or who were diagnosed with cancer, chronic renal failure, dementia, ischemic stroke, or rare/incurable diseases during the entire follow-up period $(n=2,905)$ or who had GC prescriptions for 3 months or more during the 1 year before the index date were also excluded. The final study population consisted of 1,896,159 participants who were followed up through December 31, 2009 (Fig. 1). The Institutional Review Board of ChungAng University Hospital reviewed and approved this study (IRB 
2,000,000 People had health checkups in 2006

3,759 People were under 20 or over 100

1,839 People died within 6 months since the index date

67,405 People had GC prescriptions for 3 months preceding the index date

29,051 People were diagnosed with cancer, chronic renal failure, dementia, ischemic stroke, or rare/incurable diseases during the whole follow-up period

1,186 People suffered a fracture within 6 months after the index date

601 People had no information on smoking, alcohol use, BMI, and place of residence

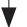

1,896,159 People met all inclusion criteria

Fig. 1. Selection of study subjects. GC, glucocorticoid; BMI, body mass index.

No. C201486 (1282)). Written informed consent by the patients was waived due to a retrospective nature of our study.

GC users were defined as patients with at least one pharmacy claim for a GC. The index date for GC users was the date of the first GC prescription claim, and the index date for non-users was the date of the checkup. The follow-up period was the 2-year period after the index date. The follow-up period for patients reporting a fracture after the index date ended on the date of fracture occurrence. For patients who did not have a fracture after the index date, the follow-up period was a minimum of 2 years and ended either when the patient died or at the end of the study period.

\section{Measurement of GC use}

We included all kinds of oral and parenteral GCs, such as deflazacort, dexamethasone, hydrocortisone, methylprednisolone, prednisolone, and triamcinolone. We considered GCs with systemic effects, but not inhaled or topical GCs. Also included were ingredients that were classified as $\mathrm{H} 02 \mathrm{AB}$ and $\mathrm{H} 02 \mathrm{BX}$ in the WHO ATC codes (2013). In addition, GC doses were calculated as prednisolone-equivalent doses. We calculated the total amount of GC used for 6 months from the index date using the DDD. Only patients who had ever taken a GC during the first 6 months were included and categorized as GC users in this anal- ysis The DDD is the assumed average maintenance dose per day of a drug used for its main indication in adults [15]. The amount of medication can be added up regardless of the ingredients or administration route. For example, if one is prescribed $5 \mathrm{mg}$ of prednisolone, which has a DDD of $10 \mathrm{mg}$, to take every day for 90 days, the total medication use would sum up to 45 DDDs. If instead, one received $10 \mathrm{mg}$ of prednisolone for 90 days, the total would be 90 DDDs. The subjects were categorized into four groups based on GC use: non-users (GC DDDs= 0 ), low users ( $0<\mathrm{GC}$ DDDs $\leq 45)$, intermediate users $(45<\mathrm{GC}$ DDDs $\leq 90)$, and high users ( $90<$ GC DDDs).

\section{Fracture incidence}

We identified all patients who had a hip or vertebral fracture recorded in their claim records during follow-up. We identified all claims records of outpatient visits or hospital admissions of patients from January 1, 2006 to December 31, 2009 in the NHIS data. We used specific International Classification of Diseases, Tenth Revision (ICD-10) codes and procedures to identify hip and vertebral fractures $[16,17]$. Hip fractures were identified by the ICD-10 codes of S72.0 (fracture of the femoral neck) and S72.1 (pertrochanteric fracture) and codes for seven procedures (open reduction of fractured extremity-femur, closed pinningfemur, external fixation-pelvis/femur, closed reduction of fractured extremity-pelvis/femur, bone traction, skin traction, hemiarthroplasty-hip). For the spine, the codes were S22.0 (fracture of the thoracic spine), S22.1 (multiple fractures of the thoracic spine), S32.0 (fracture of the lumbar spine), M48.4 (fatigue fracture of vertebra), and M48.5 (collapsed vertebra, not elsewhere classified). Each fracture code had to be accompanied by a physician's claim for site-specific fracture reduction or fixation (either open or closed) to enhance the specificity of the coding [18].

\section{Covariates}

The covariates were age, sex, place of residence, fracture history, rheumatoid arthritis history, osteoporosis treatment medication (bisphosphonates and selective estrogen receptor modulators), alcohol consumption, smoking status, and BMI. Sex, age, place of residence, alcohol consumption, smoking status, and BMI were defined at the date of the health checkups. Patients' history of fracture, osteoporosis treatment medication, and rheumatoid arthritis were also investigated in the NHIS database. A history of a fracture was defined if a vertebral or hip fracture had occurred during the 2 years before the index date. A history of rheumatoid arthritis was defined as a subject visiting an out- 
patient clinic more than twice or being admitted more than once using a related code during 1 year preceding the index date.

\section{Statistical analysis}

Most covariates, such as gender, place of residence, fracture history, rheumatoid arthritis history, alcohol consumption, smoking, and osteoporosis treatment medication, were set as dichotomous variables. BMI was classified into three groups; underweight $\left(<18.5 \mathrm{~kg} / \mathrm{m}^{2}\right)$, normal weight (18.5 to $\left.22.9 \mathrm{~kg} / \mathrm{m}^{2}\right)$, and overweight and obese $\left(\geq 23 \mathrm{~kg} / \mathrm{m}^{2}\right)$. Age was analyzed as a continuous variable. The baseline characteristics of the four GC groups were compared using the chi-square test. Cox proportional hazards regression was performed to evaluate the association between GC use and hip and vertebral fractures. The cumulative hazard rate of fracture according to GC use was depicted as a survival curve. Statistical significance was assumed at $\alpha=$ 0.05 and all analyses were conducted using SAS version 9.4 software (SAS Institute Inc., Cary, NC, USA).

\section{RESULTS}

\section{Demographic and clinical characteristics}

Table 1 summarizes the baseline characteristics of the four groups defined by total GC use. There were 1,896,159 individuals in the cohort, and the prevalence of GC use was $34.6 \%$ (656,419 subjects for all type therapies). However, except for the low user group, it was only $1.05 \%$.

The proportions of women and rural residents were higher in $\mathrm{GC}$ users than in non-users. The mean age of the subjects was 44.8 years, and it increased in groups with higher GC use. GC users were more likely to be diagnosed with fractures, osteoporosis, and rheumatoid arthritis than non-GC users. In contrast, non-GC users were more likely to be drinkers and smokers than $\mathrm{GC}$ users. The mean BMI was similar among the four groups, but the normal and overweight or obese BMI groups were proportionally more represented among $\mathrm{GC}$ users than among GC non-users.

\section{Incidence of fractures according to $\mathbf{~ G C}$ use}

Over 2 years of follow-up, 4,793 (0.25\%) individuals experienced fractures, including 3,988 vertebral fractures and 880 hip fractures (Fig. 2). Fractures were more likely in GC users than in non-GC users. For vertebral fractures, the highest incidence was found in the high-users group (80 patients per 10,000) and the lowest in the non-user group (14 patients per 10,000). The same pattern was observed for hip fractures, which increased

Table 1. Characteristic of the Study Population by Glucocorticoid Exposure

\begin{tabular}{|c|c|c|c|c|c|c|}
\hline \multirow[b]{2}{*}{ Characteristic } & \multicolumn{4}{|c|}{ Total glucocorticoid amount } & \multirow[b]{2}{*}{ Total, $\%$} & \multirow[b]{2}{*}{ Total no. } \\
\hline & $\begin{array}{c}0 \\
\text { (non-user) }\end{array}$ & $\begin{array}{c}0<\text { DDDs } \leq 45 \\
\text { (low user) }\end{array}$ & $\begin{array}{c}45<\text { DDDs } \leq 90 \\
\text { (intermediate user) }\end{array}$ & $\begin{array}{c}90<\text { DDDs } \\
\text { (high user) }\end{array}$ & & \\
\hline Female sex, $\%^{\mathrm{a}}$ & 37.63 & 48.69 & 44.32 & 37.86 & 41.39 & 784,891 \\
\hline Age, yr & $43.58 \pm 13.23$ & $46.99 \pm 13.85$ & $51.99 \pm 13.89$ & $52.68 \pm 14.09$ & & $44.82 \pm 13.56$ \\
\hline Residence (rural), $\%{ }^{\mathrm{a}}$ & 29.05 & 32.62 & 35.79 & 35.16 & 30.32 & 574,889 \\
\hline Fracture history, $\%{ }^{a}$ & 0.11 & 0.23 & 0.39 & 0.47 & 0.16 & 2,961 \\
\hline Osteoporosis treatment medication, $\%^{\mathrm{a}}$ & 0.92 & 2.25 & 3.98 & 4.40 & 1.40 & 26,513 \\
\hline RA history, $\%^{\mathrm{a}}$ & 0.84 & 2.01 & 4.82 & 5.38 & 1.27 & 24,145 \\
\hline Alcohol use, $\%^{\mathrm{a}}$ & 19.00 & 15.97 & 15.24 & 15.39 & 17.94 & 340,258 \\
\hline Smoking, $\%{ }^{\mathrm{a}}$ & 18.84 & 13.72 & 14.42 & 15.78 & 17.08 & 323,899 \\
\hline \multicolumn{7}{|l|}{ BMI, $\%{ }^{\mathrm{a}}$} \\
\hline Low weight & 4.30 & 3.82 & 3.34 & 4.18 & 4.13 & 78,344 \\
\hline Normal & 40.45 & 38.34 & 36.01 & 37.76 & 39.70 & 752,763 \\
\hline Overweight and obese & 55.25 & 57.84 & 60.66 & 58.06 & 56.16 & $1,065,052$ \\
\hline BMI, $\mathrm{kg} / \mathrm{m}^{2}$ & $23.52 \pm 3.15$ & $23.68 \pm 3.11$ & $23.85 \pm 3.08$ & $23.68 \pm 3.15$ & $23.58 \pm 3.14$ & $1,896,159$ \\
\hline Total & $1,239,840(65.39)$ & $636,129(33.55)$ & $15,076(0.80)$ & $5,114(0.27)$ & 100.0 & $1,896,159$ \\
\hline
\end{tabular}


with increasing GC usage. The vertebral fracture rate was higher than the hip fracture rate in all groups.

\section{The relationship between clinical risk factors and fracture risk}

The risk of fractures was higher in women and increased sharply with age (Table 2). Compared to normal BMI, low BMI was associated with a 1.92 times higher (hazard ratio [HR], 1.92; 95\% confidence interval [CI], 1.52 to 2.43 ) hip fracture risk, while overweight or obesity had a lower risk (HR, 0.74; 95\% CI, 0.64 to 0.85 ). Patients who had fractured had a 4.25 times higher risk of a vertebral fracture (HR, 4.25; 95\% CI, 3.65 to 4.96) and a 3.0 times higher risk of a hip fracture (HR, 3.0; $95 \%$ CI, 2.04 to 4.40 ) than their counterparts. A history of osteoporo-

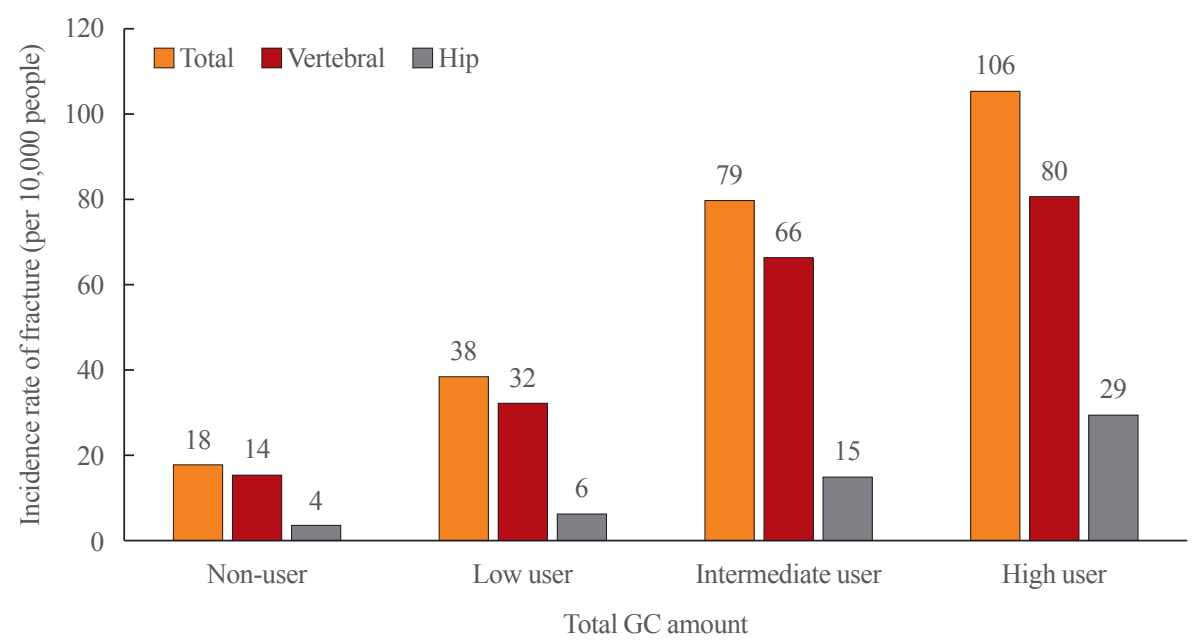

Fig. 2. Unadjusted incidence rate of vertebral and hip fracture by glucocorticoid (GC) amount the relationship between glucocorticoid exposure and fracture risk.

Table 2. Association between Glucocorticoid Amount and Fracture Risk

\begin{tabular}{|c|c|c|c|c|c|c|}
\hline \multirow{2}{*}{ Variable } & \multicolumn{2}{|c|}{ Total } & \multicolumn{2}{|c|}{ Vertebral } & \multicolumn{2}{|c|}{ Hip } \\
\hline & HR & $95 \% \mathrm{CI}$ & HR & $95 \% \mathrm{CI}$ & HR & $95 \% \mathrm{CI}$ \\
\hline \multicolumn{7}{|l|}{ Glucocorticoid amount } \\
\hline Low & 1.33 & $1.26-1.41$ & 1.39 & $1.31-1.49$ & 1.10 & $0.96-1.26$ \\
\hline Moderate & 1.88 & $1.56-2.26$ & 1.94 & $1.59-2.37$ & 1.72 & $1.12-2.64$ \\
\hline High & 2.58 & $1.97-3.38$ & 2.43 & $1.78-3.31$ & 3.28 & $1.96-5.50$ \\
\hline Female sex & 2.31 & $2.15-2.48$ & 2.68 & $2.47-2.90$ & 1.27 & $1.09-1.47$ \\
\hline Age & 1.12 & $1.11-1.12$ & 1.11 & $1.11-1.12$ & 1.13 & $1.13-1.14$ \\
\hline Residencial area (rural) & 1.33 & $1.25-1.40$ & 1.40 & $1.31-1.49$ & 0.98 & $0.86-1.13$ \\
\hline Fracture history & 4.04 & $3.50-4.66$ & 4.25 & $3.65-4.96$ & 3.00 & $2.04 \quad 4.40$ \\
\hline Osteoporosis treatment medication & 1.54 & $1.41-1.68$ & 1.59 & $1.44-1.75$ & 1.30 & $1.02-1.65$ \\
\hline RA history & 1.18 & $1.03-1.36$ & 1.14 & $0.97-1.33$ & 1.45 & $1.06-2.00$ \\
\hline Alcohol use & 1.22 & $1.10-1.35$ & 1.26 & $1.12-1.41$ & 1.16 & $0.93-1.44$ \\
\hline Smoking & 1.29 & $1.16-1.44$ & 1.32 & $1.17-1.50$ & 1.20 & $0.96-1.50$ \\
\hline \multicolumn{7}{|l|}{ BMI } \\
\hline Low weight & 1.35 & $1.19-1.53$ & 1.22 & $1.05-1.41$ & 1.92 & $1.52-2.43$ \\
\hline Normal & - & - & - & - & - & - \\
\hline Overweight and obese & 0.98 & $0.92-1.04$ & 1.03 & $0 .-1.10$ & 0.74 & $0.64-0.85$ \\
\hline
\end{tabular}



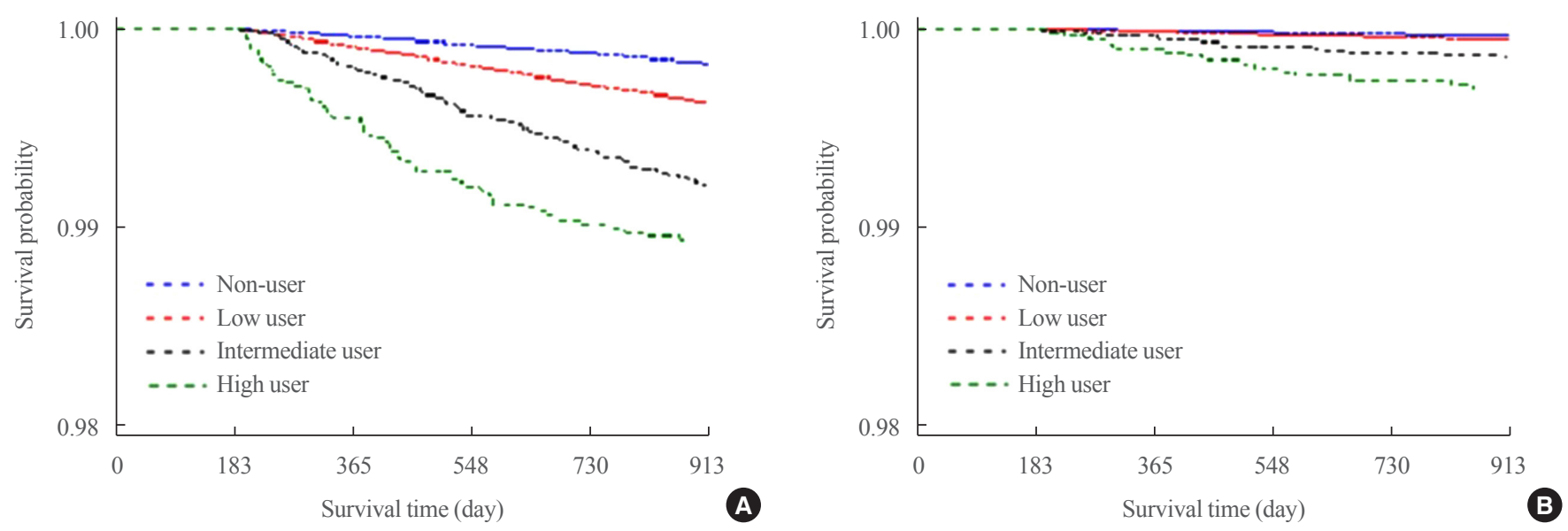

Fig. 3. Survival distribution function estimates for fracture. (A) Vertebral fracture. (B) Hip fracture.

sis treatment medication was related to a higher risk of vertebral fractures (HR, 1.59; 95\% CI, 1.44 to 1.75$)$ and hip fractures (HR, 1.30; 95\% CI, 1.02 to 1.65). In addition, smoking, alcohol consumption, and rural residency were associated with an elevated vertebral fracture risk, and a history of rheumatoid arthritis was related to a higher hip fracture risk.

\section{The relationship between GC exposure and fracture risk}

Table 2 shows associations between the amount of GC use and fracture risk according to a Cox proportional hazards model. After adjusting for the covariates, the vertebral fracture risk of $\mathrm{GC}$ users was higher than that of non-GC users. Even in the low-user group (under 45 DDDs), the vertebral fracture risk was 1.39 times higher (HR, 1.39; 95\% CI, 1.31 to 1.49 ) than among non-users. The vertebral fracture risk was 2.43 times higher (HR, 2.43; 95\% CI, 1.78 to 3.31) in the high-user group and 1.94 times higher (HR, 1.94; $95 \% \mathrm{CI}, 1.59$ to 2.37 ) in the intermediate-user group than in the non-user group, respectively. Similarly, the risk of hip fractures was higher in GC users than in non-users. The hip fracture risk was 3.28 times higher (HR, 3.28 ; $95 \%$ CI, 1.96 to 5.50 ) in the high-user group and 1.72 times higher (HR, $1.72 ; 95 \% \mathrm{CI}, 1.12$ to 2.64 ) in the intermediate-user group than in the non-user group. Fig. 3 shows how the fracture-free survival probability changed over time for each of the four GC user groups during the 2-year follow-up period, correcting for other factors. Higher amounts of GC use were associated with more vertebral fractures over time. Hip fracture also occurred more frequently with increasing GC usage over time. The risk of fractures among the four GC user groups increased over time.

\section{DISCUSSION}

In this study, we evaluated the relationship between GC usage and vertebral and hip fractures in a large population cohort of $1,896,159$ subjects who received National Health Checkups in 2006. The subjects were divided into four groups according to GC use over the course of 6 months and were followed for vertebral and hip fractures for 2 years. GC users accounted for $34.61 \%$ of the study population. Relative to non-users, the vertebral fracture risk was 1.39 times higher ( $95 \%$ CI, 1.31 to 1.49 ) even in low users. The risk of hip fractures was 1.72 times and 3.28 times higher in moderate users and high users, respectively, compared to low users. Fracture risk can be predicted by evaluating GC usage for 6 months. To our knowledge, this is the first large-scale study of this issue in Korea using the nationwide National Health Insurance claims database.

The adverse effects of systemic GCs on bone fragility are well known. Many studies have examined the relationship between GC use and fracture risk; however, several differences exist among these studies in terms of subjects and the details of GC use. Furthermore, numerous studies have analyzed the use of GCs and fracture risk in patients with diseases for which GCs are used for therapeutic purposes, such as rheumatoid arthritis, systematic lupus erythematosus, and chronic obstructive pulmonary disease [19-21]. In contrast, this study was conducted among members of the general public who underwent National Health Checkups. In previous cohort studies on the general population, the use of GCs has only been evaluated in terms of never or ever. Most studies of this issue have presented little information on dosage or duration. Kanis et al. [10] showed that prior GC use increased the relative risk of hip fracture by 2.62 
times in men and by 2.07 times in women in a previous metaanalysis of seven cohorts. Recently, the Canadian Multicenter Osteoporosis Study reported that baseline GC use affected fracture risk over the course of 10 years, irrespective of the dose and precise duration [22]. In the present study, similar results were obtained, as GC use for the first 6 months increased the risk of subsequent fractures.

The details of GC use have been presented in epidemiological studies using claims data, with subjects often divided into groups according to daily dose, duration, and pattern of use. Representatively, van Staa et al. [6] reported similar results in the GPRD in the United Kingdom to those found in our study. They showed a dose-dependent effect of oral GCs on fracture according to the daily dose. The risk of vertebral fractures increased by more than 1.5 times at low doses (less than $2.5 \mathrm{mg}$ / day), and the risk of hip fractures increased by 1.77 times and 2.27 times at medium doses ( 2.5 to $7.5 \mathrm{mg} /$ day) and high doses (7.5 mg/day or more) [6]. Since then, several studies have reported an increased risk of fractures at daily dose of GCs exceeding $5 \mathrm{mg}$. However, in most studies, the duration of GC use varied from patient to patient, which is problematic because the concept of daily doses without consideration of duration of use can be confusing when applied in practice. Therefore, recent studies have additionally analyzed the cumulative dose, peak dose, and duration [23], but the method of evaluating GC usage has not yet been standardized. To date, no clear information has been presented on the risk of fracture with long-term use of low doses of GCs. In this study, we evaluated the use of GCs on the basis of DDDs. For example, the use of $2.5 \mathrm{mg}$ of prednisolone for 6 months $(\mathrm{DDD}=45)$ significantly increased the risk of vertebral fractures. This suggests that the long-term use of lowdose GCs may be risky. In addition, this study evaluated GC use for 6 months with standardized DDDs in consideration of dose and duration, in order to provide more detailed information for use in predicting fracture risk in clinical practice.

Several studies have estimated the prevalence of oral GC use in the general population. In the United Kingdom adult population (age $\geq 18$ years), the annual prevalence of long-term (i.e., $>3$ months) oral GC prescriptions was on average $0.75 \%$ between 1989 and 2008 [7], and in the United States population aged $\geq 20$ years, the average proportion of oral GC users was $1.2 \%$ for 10 years from 1999 to 2008 [8]. In our study, 34.61\% of all subjects (aged $\geq 40$ years) had used GCs for all type therapies. However, more than intermediate users, i.e., patients exposed more than using $5 \mathrm{mg}$ prednisolone for 3 months, were $1.05 \%$. In this study, both oral and injectable drugs were includ- ed, which may have resulted in a higher percentage of GC users than reported in previous studies. In other words, it suggests the possibility of frequent uses of a short-term GC injection. Although it is not possible to directly compare usage by country, we can assume that injectable GCs are frequently used in South Korea. This suggests that the prevalence of GIOP is likely high in Korea, but fewer than $<5 \%$ of intermediate and high users received osteoporosis medication in this study. This was much lower than the proportion of patients in these groups receiving medication for general osteoporosis, and it is likely that osteoporosis was only diagnosed by BMD tests, in accordance with existing domestic insurance standards. Therefore, criteria for treatment should be established that account for the characteristics of GIOP, including most notably an increased risk of fracture before bone mass decreases.

The low-user group, with $\leq 45$ DDDs, comprised the most common GC group (33.5\%). In the low-user group, the risk of hip fracture did not increase significantly, but the risk of vertebral fracture increased by 1.39 times. This is consistent with previous findings, and can be explained by the fact that GC-induced bone loss is especially prominent in spongy bones such as the lumbar spine [24]. Therefore, even in low users, a proper assessment and treatment for fracture risk should be performed at the beginning of administration. Further research is needed to find the cut-off value of GC use that is most closely associated with increased fracture risk in low-user groups.

This study has several limitations. First, the database was based on medical claims, and therefore GC use could not be precisely measured. The exact amount of GC use could have been lower than what was prescribed, and consequently the effects of GC on fracture risk could have been underestimated. Second, as we used the DDD methodology to evaluate to total GC use, the duration of use could have been different in patients with equal DDDs. In this regard, future research should analyze whether there is a difference in fracture risk between high-dose/ short-duration use and low-dose/long-duration use. Third, as this cohort consisted of only people who received health checkups, there could have been selection bias between subjects who received health checkups and those who did not, even though the population was randomly sampled from all individuals who received health checkups in 2006. Fourth, there was no information about BMD, and therefore the baseline BMD could not be controlled. Fifth, underlying disease itself that needs GC treatment might have potential independent effects on fracture risk. In this study, only rheumatoid arthritis was included in the analysis, and further studies are needed. Finally, as we defined 
vertebral and hip fractures by diagnostic and procedure codes, we may have missed asymptomatic vertebral fractures. Therefore, fracture risk might have been underestimated due to this limitation of analyses of claims data.

In conclusion, this large-scale cohort study confirmed that the risk of hip and vertebral fractures depended on total GC use. This implies that the estimation of GC use for even 6 months can predict the risk of fractures that persists for at least 2 years even if patients discontinue GC use, and that clinicians should monitor this possibility appropriately. Further studies are needed to identify high-risk patients for fractures among those who receive low doses of GCs.

\section{CONFLICTS OF INTEREST}

No potential conflict of interest relevant to this article was reported.

\section{ACKNOWLEDGMENTS}

We acknowledge the research support from National Health Insurance Services (NHIS). The National Health Checkup database and the National Health Insurance Database were made available by NHIS. This research was also supported by a grant of the Korea Health Technology R\&D Project through the Korea Health Industry Development Institute (KHIDI), funded by the Ministry of Health and Welfare, Korea (grant number: HI13C1522).

\section{AUTHOR CONTRIBUTIONS}

Conception or design: Y.C.H., T.Y.K., Y.K.L., H.Y.K., S.J. Acquisition, analysis, or interpretation of data: J.W.K., J.K., H.C. Drafting the work or revising: J.W.K., H.Y.K. Final approval of the manuscript: H.Y.K., S.J.

\section{ORCID}

Ji Weon Koh https://orcid.org/0000-0002-5347-4053

Junkang Kim https://orcid.org/0000-0002-1967-0819

Ha Young Kim https://orcid.org/0000-0002-0651-2213

Sunmee Jang https://orcid.org/0000-0002-8771-8693

\section{REFERENCES}

1. Saag KG, Koehnke R, Caldwell JR, Brasington R, Burmeister LF, Zimmerman B, et al. Low dose long-term corticoste- roid therapy in rheumatoid arthritis: an analysis of serious adverse events. Am J Med 1994;96:115-23.

2. Lane NE, Lukert B. The science and therapy of glucocorticoid-induced bone loss. Endocrinol Metab Clin North Am 1998;27:465-83.

3. Buckley L, Humphrey MB. Glucocorticoid-induced osteoporosis. N Engl J Med 2018;379:2547-56.

4. Panday K, Gona A, Humphrey MB. Medication-induced osteoporosis: screening and treatment strategies. Ther Adv Musculoskelet Dis 2014;6:185-202.

5. LoCascio V, Bonucci E, Imbimbo B, Ballanti P, Adami S, Milani S, et al. Bone loss in response to long-term glucocorticoid therapy. Bone Miner 1990;8:39-51.

6. Van Staa TP, Leufkens HG, Abenhaim L, Zhang B, Cooper C. Use of oral corticosteroids and risk of fractures. June, 2000. J Bone Miner Res 2005;20:1487-94.

7. Fardet L, Petersen I, Nazareth I. Prevalence of long-term oral glucocorticoid prescriptions in the UK over the past 20 years. Rheumatology (Oxford) 2011;50:1982-90.

8. Overman RA, Yeh JY, Deal CL. Prevalence of oral glucocorticoid usage in the United States: a general population perspective. Arthritis Care Res (Hoboken) 2013;65:294-8.

9. Kim HY, Jang EJ, Park B, Kim TY, Shin SA, Ha YC, et al. Development of a Korean Fracture Risk Score (KFRS) for predicting osteoporotic fracture risk: analysis of data from the Korean National Health Insurance Service. PLoS One 2016;11:e0158918.

10. Kanis JA, Johansson H, Oden A, Johnell O, de Laet C, Melton III LJ, et al. A meta-analysis of prior corticosteroid use and fracture risk. J Bone Miner Res 2004;19:893-9.

11. Kanis JA, Johansson H, Oden A, McCloskey EV. Guidance for the adjustment of FRAX according to the dose of glucocorticoids. Osteoporos Int 2011;22:809-16.

12. De Vries F, Bracke M, Leufkens HG, Lammers JW, Cooper C, Van Staa TP. Fracture risk with intermittent high-dose oral glucocorticoid therapy. Arthritis Rheum 2007;56:208-14.

13. Mandel S, Schilling J, Peterson E, Rao DS, Sanders W. A retrospective analysis of vertebral body fractures following epidural steroid injections. J Bone Joint Surg Am 2013;95: 961-4.

14. Sutter SA, Stein EM. The skeletal effects of inhaled glucocorticoids. Curr Osteoporos Rep 2016;14:106-13.

15. Choi JH, Kim GA, Park JH, Song GH, Park JW, Kim DY, et al. Generation of viable embryos and embryonic stem celllike cells from cultured primary follicles in mice. Biol Reprod 2011;85:744-54. 
16. Lim S, Koo BK, Lee EJ, Park JH, Kim MH, Shin KH, et al. Incidence of hip fractures in Korea. J Bone Miner Metab 2008;26:400-5.

17. Lippuner K. Epidemiology of osteoporotic fractures in Switzerland. Rev Med Suisse 2009;5:1304-8.

18. Park C, Ha YC, Jang S, Jang S, Yoon HK, Lee YK. The incidence and residual lifetime risk of osteoporosis-related fractures in Korea. J Bone Miner Metab 2011;29:744-51.

19. Balasubramanian A, Wade SW, Adler RA, Lin CJF, Maricic M, O’Malley CD, et al. Glucocorticoid exposure and fracture risk in patients with new-onset rheumatoid arthritis. Osteoporos Int 2016;27:3239-49.

20. Bultink IE, Harvey NC, Lalmohamed A, Cooper C, Lems WF, van Staa TP, et al. Elevated risk of clinical fractures and associated risk factors in patients with systemic lupus erythematosus versus matched controls: a population-based study in the United Kingdom. Osteoporos Int 2014;25:1275-83.

21. Oshagbemi OA, Burden AM, Shudofsky KN, Driessen
JHM, Vestergaard P, Krings A, et al. Use of high-dose intermittent systemic glucocorticoids and the risk of fracture in patients with chronic obstructive pulmonary disease. Bone 2018;110:238-43.

22. Ioannidis G, Pallan S, Papaioannou A, Mulgund M, Rios L, Ma J, et al. Glucocorticoids predict 10-year fragility fracture risk in a population-based ambulatory cohort of men and women: Canadian Multicentre Osteoporosis Study (CaMos). Arch Osteoporos 2014;9:169.

23. van Staa TP, Leufkens HG, Abenhaim L, Zhang B, Cooper C. Oral corticosteroids and fracture risk: relationship to daily and cumulative doses. Rheumatology (Oxford) 2000;39: 1383-9.

24. Angeli A, Guglielmi G, Dovio A, Capelli G, de Feo D, Giannini $S$, et al. High prevalence of asymptomatic vertebral fractures in post-menopausal women receiving chronic glucocorticoid therapy: a cross-sectional outpatient study. Bone 2006;39:253-9. 